\title{
Start codon mutation of GYG1 causing late-onset polyglucosan body myopathy with nemaline rods
}

\section{Tasca, Giorgio}

2016-10

Tasca , G , Fattori , F , Monforte , M , Hedberg-Oldfors , C , Sabatelli , M , Udd , B , Boldrini , R , Bertini , E , Ricci , E \& Oldfors , A 2016 , ' Start codon mutation of GYG1 causing late-onset polyglucosan body myopathy with nemaline rods ' , Journal of Neurology, vol. 263 , no. 10 , pp. 2133-2135 . https://doi.org/10.1007/s00415-016-8268-z

http://hdl.handle.net/10138/228319

https://doi.org/10.1007/s00415-016-8268-z

publishedVersion

Downloaded from Helda, University of Helsinki institutional repository.

This is an electronic reprint of the original article.

This reprint may differ from the original in pagination and typographic detail.

Please cite the original version. 


\title{
Start codon mutation of GYG1 causing late-onset polyglucosan body myopathy with nemaline rods
}

\author{
Giorgio Tasca $^{1}$ (1) Fabiana Fattori ${ }^{2} \cdot$ Mauro Monforte $^{3} \cdot$ Carola Hedberg-Oldfors $^{4}$. \\ Mario Sabatelli ${ }^{3} \cdot$ Bjarne Udd $^{5,6,7} \cdot$ Renata Boldrini $^{8} \cdot$ Enrico Bertini $^{2} \cdot$ \\ Enzo Ricci ${ }^{3} \cdot$ Anders Oldfors $^{4}$
}

Received: 11 June 2016/Revised: 9 August 2016/Accepted: 10 August 2016/Published online: 20 August 2016

(c) Springer-Verlag Berlin Heidelberg 2016

\section{Dear Sirs,}

Polyglucosan body myopathies are a clinically and genetically heterogeneous group of muscle disorders pathologically characterized by accumulations of alpha-amylaseresistant glycogen [1]. One recently identified form of polyglucosan body myopathy (glycogen storage disease type XV) is caused by deficiency of glycogenin-1, encoded by $G Y G 1$. Glycogenin-1 is a glycosyltransferase forming the priming oligosaccharide chain and constituting a protein core of normal glycogen. The spectrum of diseases caused by glycogenin-1 deficiency ranges from the

Electronic supplementary material The online version of this article (doi:10.1007/s00415-016-8268-z) contains supplementary material, which is available to authorized users.

Giorgio Tasca

giorgiotasca81@gmail.com

1 Don Carlo Gnocchi ONLUS Foundation, Milan, Italy

2 Unit of Neuromuscular Disorders, Laboratory of Molecular Medicine, Bambino Gesu' Children's Research Hospital, Rome, Italy

3 Institute of Neurology, Catholic University School of Medicine, Largo A. Gemelli 8, 00168 Rome, Italy

4 Department of Pathology, Institute of Biomedicine, University of Gothenburg, Gothenburg, Sweden

5 Department of Medical Genetics, Folkhälsan Institute of Genetics, Haartman Institute, University of Helsinki, Helsinki, Finland

6 Neuromuscular Research Center, University of Tampere and Tampere University Hospital, Tampere, Finland

7 Neurology Department, Vaasa Central Hospital, Vaasa, Finland

8 Department of Pathology, Bambino Gesu' Children's Research Hospital, Rome, Italy originally described severe cardiomyopathy without polyglucosan bodies in skeletal muscle [2], to isolated myopathy with juvenile or adult-onset [3-7].

Our patient, an 84-year-old male, had progressive pain and weakness in the upper left arm since age 82. Later, the weakness had spread to proximal lower limbs, right upper arm and distal lower limbs. Physical examination showed waddling and stepping gait. He could raise his right arm $30^{\circ}$ and left arm $60^{\circ}$. Hypotrophy of the right biceps brachii, forearm, first dorsal interosseous, and right thigh muscles was noticed. Weakness was present in the hand finger extensor (Medical Research Council, MRC 3), tibialis anterior (MRC 3 on the right and 4 on the left), extensor hallucis longus (MRC 2), hip flexor (MRC 3 and 4) and hip extensor (MRC 2) muscles.

Family and previous medical histories were unremarkable, except for a neurosensorial hearing loss that required prosthesis at age 70. A pedigree is shown in Supplemental Figure 1. Creatine kinase level was normal. Electromyography showed myopathic recruitment together with some large amplitude motor unit potentials and spontaneous activity. No signs of cardiomyopathy were found, and a first-degree atrioventricular block was the only abnormality on electrocardiogram.

Biopsy of the left biceps brachii showed fibers depleted of glycogen and fibers with vacuoles filled with material, which stained intensely positive with periodic acid-Schiff (PAS) and was partly resistant to alpha-amylase treatment. $38 \%$ of the fibers also contained collections of nemaline rods. Electron microscopy confirmed the presence of nemaline rods and areas of myofibrillar disruption often adjacent to the PAS-positive regions, which displayed normal glycogen as well as accumulation of material compatible with polyglucosan (Fig. 1a-f). Polyglucosan bodies were also positively immunostained for desmin and sequestosome-1 (p62) (Supplemental Figure 2). Muscle 

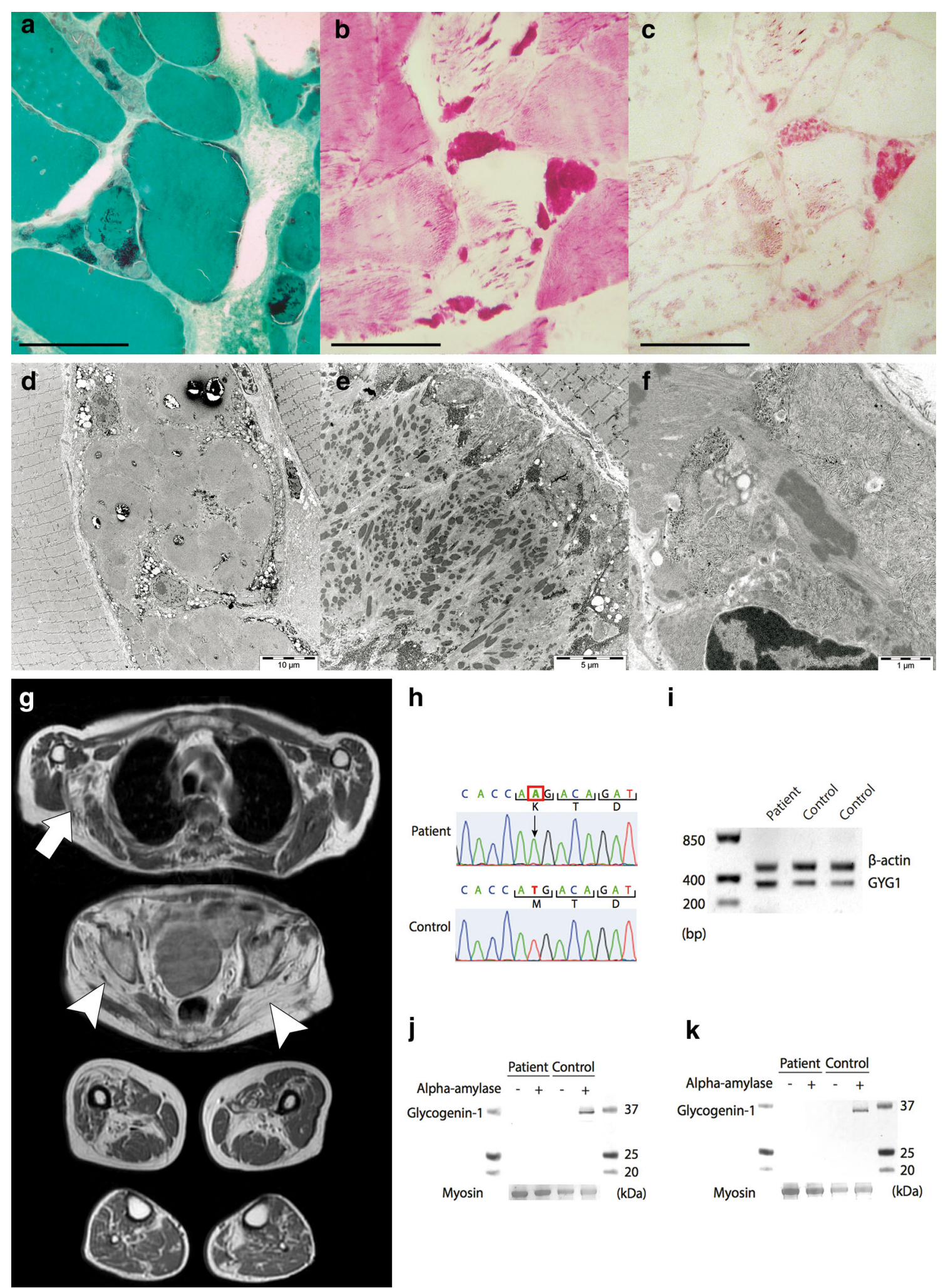

h
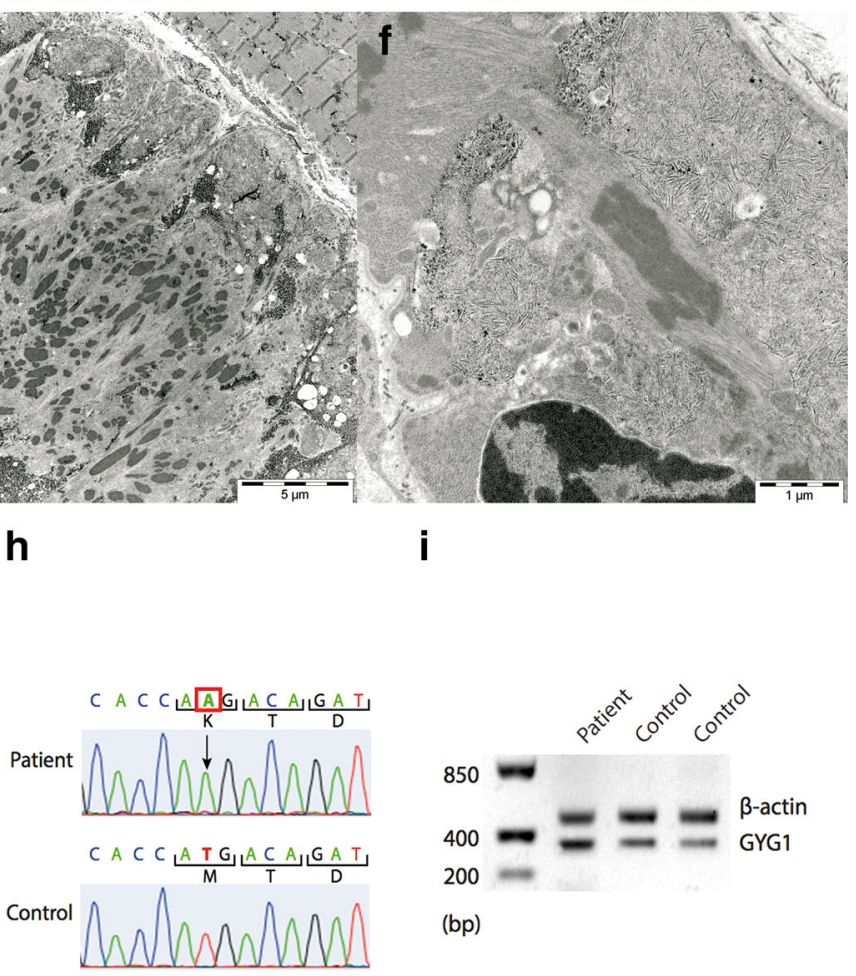

j

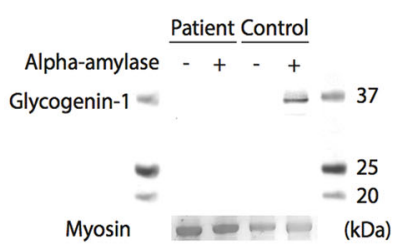

\section{k}

Alpha-amylase $\frac{\text { Patient }}{-+} \frac{\text { Control }}{-++}$

Glycogenin-1 - $\quad-37$

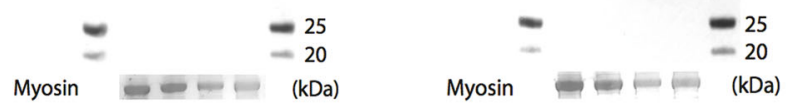


४Fig. 1 Muscle pathology, MRI and molecular studies. Gomori trichrome staining of muscle biopsy (a) showing several fibers harboring nemaline rods and vacuoles, which were intensely positive on PAS staining (b) and mostly alpha-amylase resistant (c) PAS diastase staining. Bars: 100 microns. Electron micrographs showing a fiber filled with polyglucosan bodies (d) another fiber with rods of different sizes in the central area associated with subsarcolemmal glycogen accumulations and polyglucosan bodies (e) and filamentous inclusions again in close association with rod structures (f) Other areas of less well-defined Z-disk abnormalities were present as well. (g) Muscle imaging showing fatty changes particularly in the right deltoid, infraspinatus and teres major (arrow) at the upper girdle, while glutei (arrowhead), adductor magnus, semimembranosus, biceps femoris long head, and right quadriceps (rectus femoris and vastus medialis in particular) were the most affected muscles in the pelvis and lower limbs. Focal areas of hyperintense signal on STIR sequences were also noted. (h) Electropherogram showing the homozygous missense mutation disrupting the GYG1 start codon. (i) RT-PCR on RNA from muscle tissue showing normal expression of the mutated GYG1 transcript compared to two control samples (age 28 and 53). Sanger sequencing confirmed the mutation. Western blot analyses on protein extract from muscle tissue showing absence of the glycogenin-1 with two antibodies directed against the $\mathrm{N}$ - (j) and C-terminus (k) of the protein (anti-glycogenin-1 N-terminal antibody M07 clone 3B5, Abnova, Taipei, Taiwan, dilution 1:500; C-terminal antibody HPA030497, Atlas Antibodies, Stockholm, Sweden, dilution 1:500) compared to an age-matched control sample (age 85). The use of a C-terminal antibody excluded the possibility of an alternative start codon downstream the genetic defect leading to a shorter protein missing the $\mathrm{N}$-terminus

MRI showed asymmetric and patchy changes in several muscles (Fig. 1g).

Clinical and pathology findings prompted the direct analysis of $G Y G 1$, and a homozygous c.2T $>$ A change was found by Sanger sequencing. This change is supposed to disrupt the initiation codon of the GYGl gene. Indeed, molecular analyses revealed absence of glycogenin-1 protein (Fig. 1h-k). A targeted next-generation sequencing of known and candidate muscle genes [8] excluded concomitant mutations in other disease genes including those associated with nemaline myopathy.

Common features of GYG1-related myopathy present in our patient are the asymmetric, proximal and distal muscle weakness and atrophy. On muscle imaging, glutei consistently appear as the most affected muscles in this disease, together with adductor magnus, infraspinatus and deltoid [4-6]. Our patient also showed neurosensorial hearing loss that has been reported in another GYG1-mutated patient in her 40s [4].

The presence of nemaline rods is a novel finding. Rods were particularly present close to the polyglucosan accumulations. Z-disk disruption and rod formation are possibly consequences of a perturbed protein turnover, but a definite link is yet to be found. A role of the ubiquitin-proteasome system in handling polyglucosan bodies is suggested by the immunoreactivity with p62 and ubiquitin [3] and by the existence of polyglucosan storage disorders caused by mutations in the ubiquitin ligases RBCK1 and malin [1]. Advanced age at biopsy might also have a role in the development of these abnormalities in our patient. Sporadic late-onset nemaline myopathy (SLONM) can also cause nemaline rod pathology [9]. However, no monoclonal peak was detected in our patient and one infusion of intravenous immunoglobulins $(2 \mathrm{~g} / \mathrm{kg})$ was performed without clear benefit. During follow-up, the disease had only a slow progression over a 3-year timeframe, without significant bulbar and respiratory impairment, which is another reason that makes SLONM unlikely, as well as a possible concomitant motor neuron disorder.

In conclusion, our case broadens the spectrum of glycogen storage disease XV. The late-onset phenotype and presence of apparently normal glycogen in muscle despite complete loss of glycogenin-1 indicates alternative priming of glycogen synthesis that needs to be further investigated.

Acknowledgments This study was supported by the Swedish Research Council (AO).

\section{Compliance with ethical standards}

Conflicts of interest On behalf of all authors, the corresponding author states that there is no conflict of interest.

Ethical standards All procedures performed were in accordance with the ethical standards stated in the Declaration of Helsinki.

\section{References}

1. Hedberg-Oldfors C, Oldfors A (2015) Polyglucosan storage myopathies. Mol Asp Med 46:85-100

2. Moslemi AR, Lindberg C, Nilsson J, Tajsharghi H, Andersson B, Oldfors A (2010) Glycogenin-1 deficiency and inactivated priming of glycogen synthesis. N Engl J Med 362:1203-1210

3. Malfatti E, Nilsson J, Hedberg-Oldfors C, Hernandez-Lain A, Michel F, Dominguez-Gonzalez C et al (2014) A new muscle glycogen storage disease associated with glycogenin-1 deficiency. Ann Neurol 76:891-898

4. Luo S, Zhu W, Yue D, Lin J, Wang Y, Zhu Z et al (2015) Muscle pathology and whole-body MRI in a polyglucosan myopathy associated with a novel glycogenin-1 mutation. Neuromuscul Disord 25:780-785

5. Colombo I, Pagliarani S, Testolin S, Cinnante CM, Fagiolari G, Ciscato P et al (2015) Longitudinal follow-up and muscle MRI pattern of two siblings with polyglucosan body myopathy due to glycogenin-1 mutation. J Neurol Neurosurg Psychiatry. doi:10. 1136/jnnp-2015-310553

6. Akman HO, Aykit Y, Amuk OC, Malfatti E, Romero NB, Maioli MA et al (2015) Late-onset polyglucosan body myopathy in five patients with a homozygous mutation in GYG1. Neuromuscul Disord 26:16-20

7. Fanin M, Torella A, Savarese M, Nigro V, Angelini C (2015) GYG1 gene mutations in a family with polyglucosan body myopathy. Neurol Genet 1:e21

8. Evila A, Arumilli M, Udd B, Hackman P (2015) Targeted nextgeneration sequencing assay for detection of mutations in primary myopathies. Neuromuscul Disord 26:7-15

9. Chahin N, Selcen D, Engel AG (2005) Sporadic late onset nemaline myopathy. Neurology 65:1158-1164 